\title{
Quantification of the VUV radiation in low pressure hydrogen and nitrogen plasmas
}

\author{
U Fantz $^{1,2}$, S Briefi ${ }^{2}$, D Rauner ${ }^{1,2}$ and D Wünderlich ${ }^{1}$ \\ ${ }^{1}$ Max-Planck-Institut für Plasmaphysik, Boltzmannstr. 2, 85748 Garching, Germany \\ ${ }^{2}$ AG Experimentelle Plasmaphysik, Universität Augsburg, Universitätsstr. 1, 86159 \\ Augsburg, Germany \\ E-mail: ursel.fantz@ipp.mpg.de
}

\begin{abstract}
Hydrogen and nitrogen containing discharges emit intense radiation in a broad wavelength region in the VUV. The measured radiant power of individual molecular transitions and atomic lines between $117 \mathrm{~nm}$ and $280 \mathrm{~nm}$ are compared to those obtained in the visible spectral range and moreover to the RF power supplied to the ICP discharge. In hydrogen plasmas driven at $540 \mathrm{~W}$ of RF power up to $110 \mathrm{~W}$ are radiated in the VUV, whereas less than $2 \mathrm{~W}$ is emitted in the VIS. In nitrogen plasmas the power level of about $25 \mathrm{~W}$ is emitted both in the VUV and in the VIS. In hydrogen-nitrogen mixtures, the NH radiation increases the VUV amount. The analysis of molecular and atomic hydrogen emission supported by a collisional radiative model allowed determining plasma parameters and particle densities and thus particle fluxes. A comparison of the fluxes showed that the photon fluxes determined from the measured emission are similar to the ion fluxes, whereas the atomic hydrogen fluxes are by far dominant. Photon fluxes up to $5 \times 10^{20} \mathrm{~m}^{-2} \mathrm{~s}^{-1}$ are obtained, demonstrating that the VUV radiation should not be neglected in surface modifications processes, whereas the radiant power converted to VUV photons is to be considered in power balances. Varying the admixture of nitrogen to hydrogen offers a possibility to tune photon fluxes in the respective wavelength intervals.
\end{abstract}

PACS: 52.25.Tx, 52.70.Kz, 52.80.Pi, 81.65.-b

Submitted to: Plasma Sources Science and Technology

\section{Introduction}

Low pressure hydrogen or nitrogen plasmas emit intense molecular and atomic radiation in a broad wavelength region in the vacuum ultraviolet spectral range (VUV: $100-200 \mathrm{~nm}$ ) and in the ultraviolet spectral range (UV: $200-400 \mathrm{~nm}$ ). In contrast to the radiation in the visible spectral range (VIS: $400-800 \mathrm{~nm}$ ) the energy of the UV/VUV photons (of the order of ten eV) is much higher. Due to the fact that the resonant transitions of molecules and atoms are often in the VUV, the VUV emission can attain a remarkable amount of the power used for plasma generation and the corresponding photon fluxes onto surfaces can become relevant for consideration in material processing and surface treatment processes. As a consequence, quantification and even control of VUV photons became desirable as summarized recently for low pressure plasmas in material processing applications [1]. Depending on the plasma process, VUV photons need to be avoided as they can damage films [2,3] or charge surfaces [4] in microelectronics processing. Polymer treatment is an example in which the effect of VUV photons can vary from undesirable to desirable [5]. Such assessments depend also on the photon flux compared to the ion flux. Furthermore, synergistic effects appear as demonstrated for example in photoresist treatment [6]. From the more fundamental point of view, additional electrons 
produced by photoelectron emission might be necessary to be considered in formation of the plasma sheath due to their contribution to the space charge [7,8]. Photon induced or photon enhanced etching are examples in which VUV photons are required and the quantities of photon fluxes and photon energy are even used for process optimization. For identification of the role of UV/VUV photons in plasma sterilization quantification of the photon fluxes in their respective wavelength range is also a necessity $[9,10]$. In order to characterize the relevance of the photon fluxes in plasma surface interaction, the quantification of the photon fluxes needs to be accompanied by the quantification of the particle fluxes onto the surface, namely the ion fluxes and the fluxes of the radicals. Special attention needs to be taken to possible synergistic effects of the three particle species (photon, ions and radicals) onto a surface. Such investigations might support the optimization of the desired plasma process, as at present the particle fluxes are often the only control parameters considered in plasma processing.

A thorough analysis of photon fluxes in the VUV of argon plasmas in an ICP has been carried out by comparing results from an emission model with measurements of the emission in the VUV and in the VIS [11]. For the measurements of the two resonance lines of argon (at 104.8 and $106.7 \mathrm{~nm}$ ) a calibrated VUV photodiode $(18-122 \mathrm{~nm})$ has been used. A good agreement between the measurements and the predictions from an emission model has been found, highlighting the relevance of radiation trapping. The photon fluxes are in the order of $10^{20}$ to $10^{21} \mathrm{~m}^{-2} \mathrm{~s}^{-1}$ and reveal a maximum with pressure and an increase with power. The ion fluxes have been estimated to be in the same order of magnitude, but depending on power, the photon flux exceeds the ion flux. The resonance lines of other rare gases have been investigated as well [12] and it has been found that the principal resonance lines typically account for $85 \%$ of the total VUV emission. First investigations to characterise Armolecular gas mixtures using oxygen, nitrogen and hydrogen are reported as well as such mixtures are often used in plasma processing. Significant contributions of the Lyman- $\alpha\left(\mathrm{L}_{\alpha}\right)$ line in hydrogen mixtures and the atomic oxygen lines in oxygen mixtures of up to $4 \%$ and $30 \%$, respectively have been found. For identification of the individual contributions a filter technique has been utilized. Beside the photodiode a sodium salicylate detector is used allowing for extending the wavelength range to $200 \mathrm{~nm}$, but only with very limited spectral resolution. Investigations of the power radiated in the VUV from a filament-driven multi-cusp arc discharge in hydrogen revealed that about $15-30 \%$ of the discharge power is dissipated via light emission in spectral range of $120-250 \mathrm{~nm}$, highlighting the relevance of the molecular emission [13].

The investigations presented in this paper are focussed on a detailed analysis of the VUV-UV-VIS radiation of molecular plasmas in an ICP (hydrogen and nitrogen plasmas) with the goal to determine the radiant power and photon fluxes in the individual wavelength ranges. Special attention is paid to identify the dominant molecular transitions contributing to the VUV-UV photon fluxes. For that purpose spectral resolved measurements are carried out using intensity calibrated VUV spectroscopy together with optical emission spectroscopy (OES) such that the wavelength range from $117-800 \mathrm{~nm}$ is covered. For hydrogen plasmas the measurements are supported by a collisional radiative model for atomic and molecular hydrogen, allowing the determination of plasma parameters such that particle fluxes can be derived as well. Ro-vibrationally resolved simulations of molecular hydrogen spectra based on a corona model are performed to obtain scaling factors for the calculation of the full band system emission from a measured wavelength interval.

The paper is organised as follows: section 2 introduces the experimental setup and the methods applied to derive the quantities of radiant power and photon fluxes. Section 3 focusses on hydrogen plasmas: measured spectra and results for the radiant power and photon fluxes in certain wavelength intervals are presented. Furthermore, the analysis of the plasma parameters is described and the resultant particle fluxes of atomic hydrogen and hydrogen ions onto a surface are compared to the photon fluxes. Section 4 is dedicated to pure nitrogen plasmas. A comparison of hydrogen and nitrogen plasmas is given in section 5 together with investigations of gas mixtures of hydrogen and nitrogen. All measurements are carried out at a fixed RF power of $540 \mathrm{~W}$ covering the pressure range from $1 \mathrm{~Pa}$ to $6 \mathrm{~Pa}$.

\section{Experimental setup and diagnostic methods}

\subsection{Experiment}

As illustrated in figure 1 the experiment consists of a quartz glass cylinder with a length of $40 \mathrm{~cm}$ and a diameter of $10 \mathrm{~cm}$. For ICP coupling a helical antenna with five windings and a total length of $7 \mathrm{~cm}$ 
is used. The antenna is connected via a matching network to an RF generator operating at a frequency of $13.56 \mathrm{MHz}$. The gases are supplied by calibrated flow meters, one for hydrogen and one for nitrogen. Typical flow rates are at about $5 \mathrm{sccm}$. The combination of a turbo pump with a roughing pump allows achieving background pressures of better than $10^{-6}$ mbar. For hydrogen plasma generation, the lowest achievable pressure is limited by the maximal available RF power of $600 \mathrm{~W}$; the maximum pressure is limited by the occurrence of discharges to peripheral vacuum components. These limitations lead to an accessible pressure range from $1 \mathrm{~Pa}$ to $10 \mathrm{~Pa}$ for this setup. In this pressure range and for all the gases under investigation, a reflected power $60 \mathrm{~W}( \pm 5 \mathrm{~W})$ is obtained at the generator for a forward power of $600 \mathrm{~W}$. Hence, $540 \mathrm{~W}$ are delivered to the setup which is quoted as the RF power in the following.

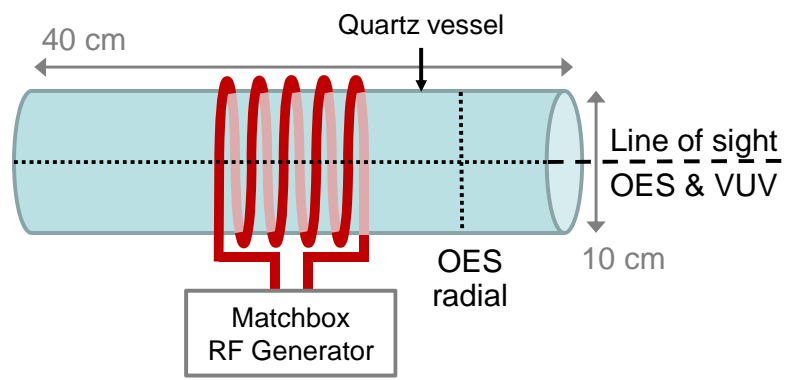

Figure 1. Sketch of the ICP with the lines of sight for VUV and OES spectroscopy.

One side of the ICP discharge is connected directly to the differentially pumped VUV spectrometer with the possibility to separate the two vacuum systems by a shutter valve. On the opposite side a quartz window is installed which provides access for optical emission spectroscopy. Both spectrometers are aligned such that they are axially centred with the advantage of having the same line of sight. The quartz vessel enables OES measurements in radial direction as well.

\subsection{Diagnostics and calibration}

For optical spectroscopy a high resolution spectrometer (Acton SP2750 system in Czerny-Turner arrangement with a focus length of $0.75 \mathrm{~m}$ and a grating with $1800 \mathrm{l} / \mathrm{mm}$ ) with a CCD camera is used. The apparatus profile is Gaussian with a FWHM of about $20 \mathrm{pm}$, depending on the wavelength. The plasma light is focussed by a lens head (quartz lens) into a UV-enhanced fibre which is connected to the entrance slit of the spectrometer. The system is wavelength calibrated by using several lamps $(\mathrm{Hg}$, $\mathrm{Hg} / \mathrm{Ar}$ and $\mathrm{Ne}$ lamps). For the calibration of the intensity of the entire spectroscopic system in the wavelength region $380-800 \mathrm{~nm}$ an absolutely calibrated Ulbricht sphere is used. By using a relatively calibrated deuterium arc lamp $(190-400 \mathrm{~nm})$ the intensity calibration is extended down to $250 \mathrm{~nm}$ taking advantage of the overlap region $380-400 \mathrm{~nm}$. The line of sight has a diameter of $1 \mathrm{~cm}$ and the discharge length of $40 \mathrm{~cm}$ is used for axial measurements to obtain the spectra in units of $\mathrm{ph} / \mathrm{nm} / \mathrm{s} / \mathrm{m}^{3}$.

The spectroscopic system for the VUV region covers the wavelength range of $105-300 \mathrm{~nm}$ consisting of a $1 \mathrm{~m}$ McPherson spectrometer (Model 225) and a solar-blind photo multiplier (EMR 51F-08-18). The grating has $12001 / \mathrm{mm}$ such that Gaussian line profiles are observed with a FWHM of about $37 \mathrm{pm}$. An aperture before the entrance slit limits the solid angle. A shutter is used such that the system can be pumped down separately to pressures below $10^{-6}$ mbar. An opening of the shutter results in an additional pumping of the discharge, such that the discharge pressure has to be readjusted to the desired value measured with a capacitive manometer. Additionally a second aperture (diameter: $4.5 \mathrm{~mm}$ ) has been introduced to limit the diameter of the line of sight to about $1 \mathrm{~cm}$. The wavelength calibration was performed using an Hg lamp to fix the $253.6 \mathrm{~nm}$ point and the zero order. A relative intensity calibration in the wavelength region of $190-300 \mathrm{~nm}$ is achieved by mounting the deuterium arc lamp directly in front of the aperture. Additionally the entire system is relatively calibrated from $117-200 \mathrm{~nm}$ by using the branching ratio method for nitrogen molecules extended by atomic nitrogen lines [14]. For that purpose nitrogen discharges are ignited in the ICP. An absolute calibration is achieved by utilizing the helium lines in the wavelength region between $270 \mathrm{~nm}$ and $300 \mathrm{~nm}$ which are detected by both the VUV system and the calibrated OES system in the radially centred axial line of sight. Generally it should be noted that the usage of the photomultiplier requires temporally stable discharges as it takes up to 20 min to scan the whole wavelength region with adequate time steps. The 
plasma stability is monitored by a survey spectrometer (axial line of sight) tracing the most intense lines in the optical wavelength range. The latter is of particular importance for hydrogen plasmas because the recombination of atomic hydrogen particles at quartz surfaces needs to achieve equilibrium first.

\subsection{Emissivity, radiant power and photon fluxes}

The absolute calibrated spectroscopic systems allow to measure the spectral intensity (spectral emissivity) in units of $\mathrm{ph} / \mathrm{nm} / \mathrm{s} / \mathrm{m}^{3}$. By integrating the spectrum over the line profile in case of atomic lines or over a certain wavelength interval for molecular emission the corresponding emissivity is obtained $\left(\mathrm{ph} / \mathrm{s} / \mathrm{m}^{3}\right)$. For the determination of the radiant power, the spectral emissivity is converted into the spectral radiant power density by using the photon energy. An integration over the wavelength interval of interest results in the radiant power density which is converted into the radiant power by considering the plasma volume of 2.77 litres. An averaged photon flux onto a surface is obtained from the emissivity by taking into account the volume to surface ratio, resulting in a conversion factor of $0.021 \mathrm{~m}$ for the present discharge geometry.

The basic assumption for these conversions is the spatial homogeneity of the plasma radiation. A first prerequisite is the homogeneity along the axial line of sight. This has been investigated by using the OES system in radial direction with several positions of the optical head along the length of the cylinder. The Balmer $\mathrm{H}_{\beta}$ line is used as a reference line as well as a molecular line of the Fulcher transition $(\mathrm{d}-\mathrm{a})$ of the hydrogen molecule; for both lines self-absorption being negligible. In all discharges investigated here, the plasma fills the whole volume. Variations of the intensity along the axial line of sight are intrinsically considered in the line of sight integrated measurements and are thus representative for the discharge.

The radial emission profile has been investigated by measuring the radial intensity profile. In order to avoid an overlap of the intensities taken at the individual spatial steps in radial direction an aperture with a diameter of $2 \mathrm{~mm}$ has been placed in front of the lens head. 27 measurement points in radial direction have been used with a step width of $3.5 \mathrm{~mm}$. Figure 2 shows the result for the $\mathrm{H}_{\beta}$ line with the intensity normalized to the maximum. The measurement has been taken at a position close to the antenna and is representative for all investigated positions along the cylinder. The corresponding emission profile is derived via solving the inverse Abel equation for the cylindrical geometry by applying the Hankel-Fourier method [15]. According to the perquisites of an Abel inversion the measured profile has been symmetrised first, normalized and the zero-position at the edges have been fixed. Nevertheless the outcome of the emission profile is very sensitive to the measured intensity profile and small deviations result in fluctuations of the emission profile. Thus, the emission profile shown in figure 2 can be regarded within the error bars as a flat emission profile.

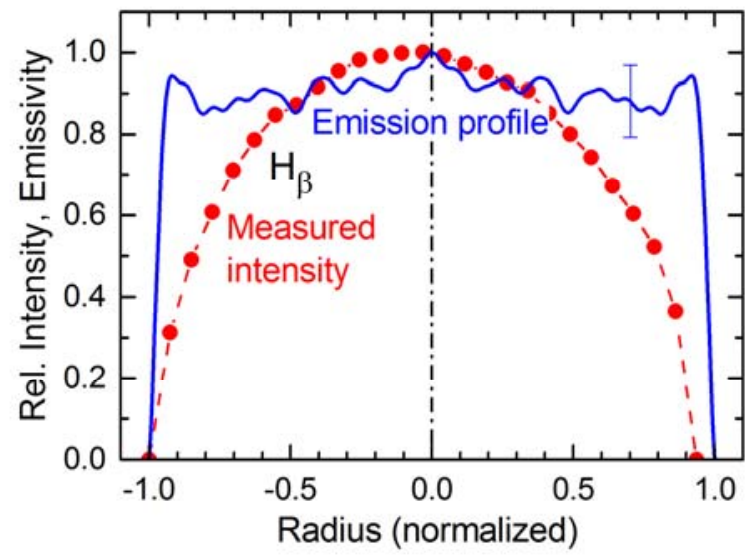

Figure 2. Measured intensity of the Balmer $\mathrm{H}_{\beta}$ line in radial direction and the corresponding emission profile after Abel inversion for a hydrogen discharge at $1 \mathrm{~Pa}$ and $540 \mathrm{~W}$ RF power.

The flat emission profile of the Balmer $\mathrm{H}_{\beta}$ line shown in figure 2 has been also observed for the molecular emission line of the Fulcher transition. Both together justify the assumption of having homogeneous distribution of the emission in radial direction within the ICP. The investigations have 
been carried out for all the pressures presented in the following. Consequently, the conversion between the quantities of emissivity and photon fluxes as well as radiant power seems to be reasonable.

Particle fluxes can be derived from particle densities which are obtained from the OES but only for hydrogen plasmas using the molecular Fulcher emission and the first four Balmer lines (see section 3.2.). In mixtures with nitrogen the Fulcher emission is partly overlapped with molecular nitrogen emission. Additionally, the Balmer lines $\mathrm{H}_{\gamma}$ and $\mathrm{H}_{\delta}$ become too weak for a thorough analysis.

\section{Hydrogen plasma}

Besides the Balmer line radiation which contributes to the radiant power and to the photon flux in the visible spectral range, the Lyman line $\mathrm{L}_{\alpha}$ is observed that contributes to the VUV emission. The other Lyman lines are not accessible by the present VUV system. From the molecule the Fulcher emission is the most intense one in the visible range, whereas several electronic transitions are prominent in the VUV-UV range.

\subsection{Molecular transitions}

Molecular radiation of an electronic transition covers a wide spectral range of several tenths of nanometres. Vibrational bands are easily resolved and due to the light mass of the molecule the individual rotational lines can often be assigned well. Figure 3 shows an overview of the electronic states in molecular hydrogen with the spectroscopic notation assigned. They split into the singlet system and the triplet system. The ground state $\mathrm{X}$ is in the singlet system. The energetically lowest state in the triplet system is the repulsive b-state covering a broad energy range (see e.g. [16] for the corresponding potential curves). The optically allowed transitions from $n=3$ and $n=2$ are indicated in figure 3 together with a classification into the dominant wavelength region for each transition.

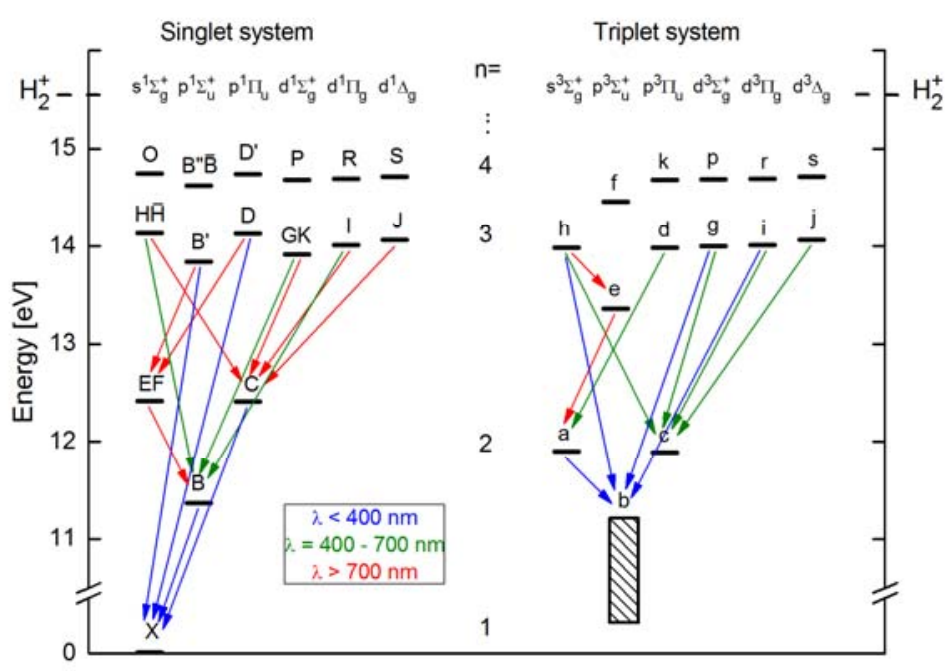

Figure 3. Energy level diagram of the electronic states of molecular hydrogen with the optically allowed transitions up to the main quantum number $\mathrm{n}=3$.

The most intense radiation of molecular hydrogen in the VUV originates from the resonant B-X and C-X transitions in the singlet system, the Lyman and Werner band respectively. The dominant part of the emission for the Lyman band is in the wavelength region of $130-170 \mathrm{~nm}$ with photon energies of $9.5-7.3 \mathrm{eV}$. The Werner band has the maximum emission typically below $130 \mathrm{~nm}$, i.e. photon energies above $9.5 \mathrm{eV}$. In the triplet system the $\mathrm{a}-\mathrm{b}$ transition is dominant, ranging from about $120 \mathrm{~nm}$ to $600 \mathrm{~nm}$. As the lower electronic state is repulsive, a broad continuum radiation is emitted. The continuum radiation overlaps in the VUV with the Lyman band. Typically the wavelength region from $190 \mathrm{~nm}(6.2 \mathrm{eV})$ to $300 \mathrm{~nm}(4.1 \mathrm{eV})$ is assigned to the continuum radiation.

\subsection{Evaluation of plasma parameters and photon fluxes by applying collisional radiative models}

The plasma parameters are derived from the emissivity of the Balmer lines $\mathrm{H}_{\alpha}-\mathrm{H}_{\delta}$ and emission of the Fulcher transition ( $\mathrm{d}-\mathrm{a})$ using the first four diagonal vibrational bands $\left(\mathrm{v}^{\prime}=\mathrm{v}^{\prime \prime}=0,1,2,3\right)$ in the 
wavelength range $600-640 \mathrm{~nm}$. Rotational lines of the Q-branch in each vibrational band are analysed such that beside the rotational population also the vibrational population is obtained [17].

The spectroscopic analysis is supported by collisional radiative (CR) models for hydrogen: for interpretation of the atomic line radiation Yacora $\mathrm{H}$ [18] is applied whereas Yacora $\mathrm{H}_{2}[19]$ is used for interpretation of the Fulcher emissivity. In case of atomic radiation the population of the upper electronic level of a Balmer line emission is in principle determined by five particle species [20]: $\mathrm{H}$ via direct excitation, $\mathrm{H}^{+}$via recombination, $\mathrm{H}_{2}$ via dissociative excitation, molecular ions $\left(\mathrm{H}_{2}^{+}\right.$and $\left.\mathrm{H}_{3}{ }^{+}\right)$ via dissociative recombination, and $\mathrm{H}^{-}$via mutual neutralisation (with $\mathrm{H}^{+}$and $\mathrm{H}_{2}{ }^{+}$). Subsequently, the $\mathrm{CR}$ model Yacora $\mathrm{H}$ is coupled to all these densities using the corresponding population coefficients [18]. Thus, line radiation depends beside the electron density and temperature also on the densities of these particle species. However, three-body and radiative recombination via $\mathrm{H}^{+}$is very unlikely in these low pressure discharges due to electron densities well below $10^{19} \mathrm{~m}^{-3}$. Mutual neutralisation of positive ions with $\mathrm{H}^{-}$is relevant only in ion sources dedicated to produce negative ions in a large amount [21]. In case of molecular hydrogen, the CR model resolves the electronic states up to the main quantum number $\mathrm{n}=3$ (indicated in figure 3). Vibrational and rotational levels are not implemented in the model. As discussed in reference [20] two data sets for the cross sections are available. For the analysis presented here the Janev data set [22] is used. At the low pressures investigated it is assumed that quenching is negligible. Another second order effect is opacity which is not relevant for the molecular radiation as the transition probability of an individual molecular line is too small but is of relevance for the atomic radiation, in particular for the Lyman series. Balmer line radiation can be treated as optically thin, however opacity of Lyman lines contributions via population escape factors also to the population of the electronic states with the consequence to increase the Balmer line radiation in such cases [23]. In order to avoid too many open parameters, the analysis is done for the optical thin case in a first step. As measurements of the electron energy distribution function are not available at the moment, the reasonable assumption of having a Maxwellian distribution is applied for the analysis.

The emissivity of the Balmer lines and the Fulcher transition from the CR models is fitted to the measurements by using the electron density, electron temperature and the atomic-to-molecular density ratio as fit parameters. The dissociative recombination process via $\mathrm{H}_{2}{ }^{+}$contributes more to the population of the higher quantum numbers of the atom such that $\mathrm{H}_{\delta}$ line is more sensitive to the $\mathrm{H}_{2}{ }^{+}$ density than $\mathrm{H}_{\gamma}$ and $\mathrm{H}_{\beta}$ and so on. The $\mathrm{H}_{2}{ }^{+}$density can be determined reasonably well to a value of $50 \%$ to $70 \%$ of the electron density. As dissociative recombination via $\mathrm{H}_{3}{ }^{+}$populates predominantly the $\mathrm{n}=2$ level, the Balmer line radiation reveals to be insensitive to the $\mathrm{H}_{3}{ }^{+}$density which is set to be at maximum the value of the electron density used for the fit. Three-body recombination and radiative recombination do also not influence the Balmer line radiation for $\mathrm{H}^{+}$densities lower than the electron density used in the fit. Hence, $\mathrm{H}^{+}$and $\mathrm{H}_{3}{ }^{+}$density are set to fulfil quasineutrality with the electron density. Consequently the $\mathrm{H}$ density, the $\mathrm{H}_{2}$ density and the $\mathrm{H}_{2}{ }^{+}$density are besides the electron temperature and density the sensitive fit parameters for the Balmer lines, whereas for the Fulcher emission the $\mathrm{H}_{2}$ density is the only relevant species. With knowledge of the gas temperature, which is derived from the rotational temperature of the Fulcher transition, the particle densities of atomic and molecular hydrogen are set to satisfy the general gas law. With the uncertainty of $10 \%$ assigned to the measured emissivities, the particle densities of $\mathrm{H}$ and $\mathrm{H}_{2}$ are determined with an error of $20 \%$, which is also the error for the electron density. The precision of the electron temperature is $\pm 0.1 \mathrm{eV}$.

The particle flux of atomic hydrogen to a surface $\Gamma_{\mathrm{H}}$ is derived from the respective particle density $\mathrm{n}_{\mathrm{H}}$ and the mean velocity: $\Gamma_{\mathrm{H}}=\mathrm{n}_{\mathrm{H}} \times \mathrm{v}_{\text {mean }} / 4$. For the atomic hydrogen temperature the gas temperature is used with the underlying assumption of having enough collisions such that the heavy particle species thermalize among each other. The ion flux is determined by the acoustic velocity: $\Gamma_{\text {ion }}=n_{\text {ion }} \times \mathrm{c}_{\mathrm{s}} / 2$ which depends on the electron temperature. The mass of $\mathrm{H}_{2}^{+}$particles is used to represent the average ion mass.

\subsection{Simulation of molecular spectra using a corona model}

Vibrationally and rotationally resolved simulations of molecular spectra allow on the one hand a distinct assignment of the individual rotational lines and vibrational bands which is in particular important when the transitions strongly overlap as it is the case for the Werner band, the Lyman band and the continuum radiation. On the other hand the emissivity of the whole band system can be 
derived from a certain wavelength interval by obtaining the corresponding scaling factors from the simulation.

The simulation of ro-vibrationally resolved molecular spectra requires besides the corresponding energy levels, the cross sections for population and depopulation processes ro-vibrationally resolved. The appropriate model would be a CR model for which the number of energy levels and thus the complexity becomes enormous. The more crucial point however, is the lack of a consistent data set for the ro-vibrationally resolved cross sections. In order to reduce the number of levels involved, the corona model is applied in which the excitation of the upper electronic state takes place from the ground state via electron impact collisions. The dominant de-excitation channel is spontaneous emission. The rotational and vibrational population of the energy levels in the ground state is characterized by a rotational temperature $\mathrm{T}_{\text {rot }}$ and a vibrational temperature $\mathrm{T}_{\text {vib. }}$. All 14 vibrational levels in the ground state of $\mathrm{H}_{2}$ are taken into account. Vibrationally resolved transitions probabilities are taken from reference [24]; the rotationally resolved transition probabilities are calculated using the LEVEL-code [25]. Ro-vibrationally resolved cross sections have been generated based on electronically resolved data ([26] for the Lyman and Werner transition in the singlet system and the data from Janev [22] for the continuum and the Fulcher band in the triplet system) using the Gryzinski method [27]. A more detailed description on the simulation of these spectra is given in reference [28]. The simulation of the continuum emission implies the consideration of a repulsive state and thus wavelength dependent transition probabilities assigned to the ro-vibrational quantum number of the upper state. Simulation of the continuum emission is applied successfully at low temperature plasmas for plasma diagnostic purposes; in particular, it has been shown that the vibrational population determines the shape of the continuum emission, whereas the intensity is sensitive to the electron density and temperature $[29,30]$.

\subsection{Example spectra and wavelength ranges}

Figure 4 shows the spectrum of a hydrogen plasma at a pressure of $3 \mathrm{~Pa}$ measured with the VUV spectrometer. In the plotted wavelength interval the Lyman band and the Werner band overlap; the wavelength ranges which are representative for the corresponding band system are indicated. The $\mathrm{L}_{\alpha}$ line lies within the Werner band and is by far the most intense line.

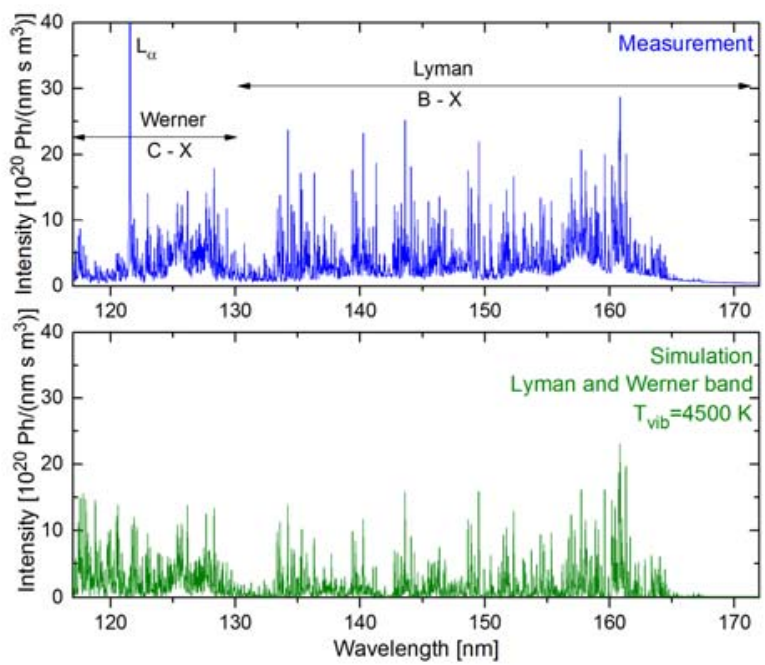

Figure 4. (a) Spectrum of a hydrogen plasma at $3 \mathrm{~Pa}$ and $540 \mathrm{~W}$ RF power. The electronic transitions are indicated. (b) Simulation of the spectrum using the corona model with the respective plasma parameters from table 1 except $\mathrm{T}_{\text {vib }}$.

In the simulation of the spectrum based on the corona model the Werner transition and the Lyman transition are taken into account. Thus, the $\mathrm{L}_{\alpha}$ line is missing in the simulated spectrum. The relative intensity is adjusted by using the vibrational and rotational temperatures as variables. The best fit is obtained for $\mathrm{T}_{\mathrm{vib}}=4500 \mathrm{~K}$ and $\mathrm{T}_{\mathrm{rot}}=720 \mathrm{~K}$ for both transitions. The band structures are clearly represented in the simulation. The absolute intensity is calculated using the electron density and temperature derived from the spectroscopic analysis supported by the CR model (described in section 
3.2 with the results presented in section 3.5, table 1). The comparison of the two spectra reveals that the decay of the envelope of the Lyman band towards lower wavelength is stronger as in the measurements. Hence, it can be concluded that additional population channels contribute to the population of the excited states like cascading from upper electronic states, i.e. from $n=3$ as shown in figure 3. Cross sections for excitation of $\mathrm{n}=3$ are in the same order of magnitude as the ones for the B- and C-states [26]. Consequently, the vibrational population in these states is also disturbed compared to the one obtained with the corona model, explaining the different decay of the envelope of the Lyman band. The latter is also supported by the need of the enhanced vibrational temperature of $\mathrm{T}_{\mathrm{vib}}=4500 \mathrm{~K}$ compared to the one obtained by the CR analysis (table $1, \mathrm{~T}_{\mathrm{vib}}=3000 \mathrm{~K}$ ). The rotational temperature however is in agreement with the gas temperature (table $1, \mathrm{~T}_{\text {gas }}=720 \mathrm{~K}$ ).

The corona spectra of the individual transitions are plotted in figure 5 using the same plasma parameters (table 1, 3 Pa case) for all three spectra except for $T_{\text {vib. }}$. The Werner system $\left(T_{\text {vib }}=4500 \mathrm{~K}\right)$ is most intense in the wavelength region $90-130 \mathrm{~nm}$ and the dominant emission of the Lyman system $\left(\mathrm{T}_{\mathrm{vib}}=4500 \mathrm{~K}\right)$ is within the wavelength region $90-165 \mathrm{~nm}$. Hence, the Lyman band overlaps the Werner band in its entire wavelength interval, whereas spectra with wavelengths higher than $130 \mathrm{~nm}$ (but lower than $190 \mathrm{~nm}$, see discussion below) can be attributed solely to the Lyman band.

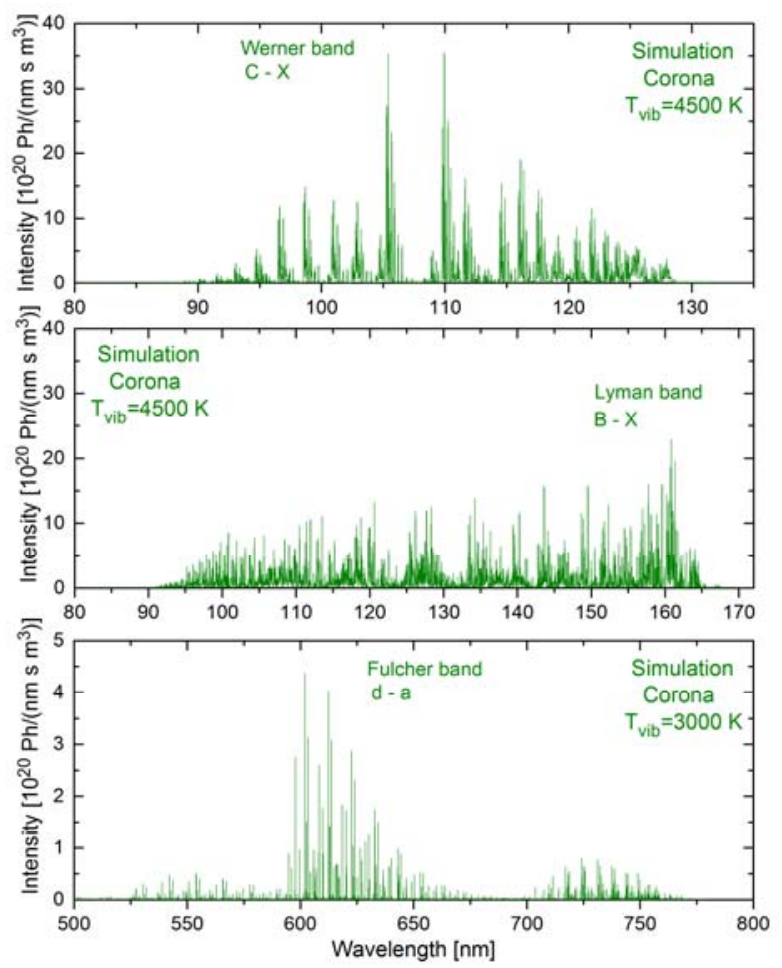

Figure 5. Simulation of the spectra of the (a) Werner band, (b) Lyman band, and (c) Fulcher band based on the corona model using the plasma parameters from table 1, except for $\mathrm{T}_{\mathrm{vib}}$, for $3 \mathrm{~Pa}$.

The Fulcher band in the triplet system of the hydrogen molecule emits in a very broad wavelength range in the VIS $(500-800 \mathrm{~nm})$. The most intense diagonal vibrational bands $\left(v^{\prime}=v^{\prime \prime}\right)$ are between $590 \mathrm{~nm}$ and $650 \mathrm{~nm}$, the non-diagonal bands extending the spectra to lower and higher wavelengths. Compared to the spectral intensity of the Werner and Lyman band, the Fulcher emission is roughly one order of magnitude lower. This is attributed to the lower excitation cross section for optically forbidden transitions, i.e. the ground state $(\mathrm{X})$ to the triplet state (d). Furthermore, higher excitation energy is required: the upper state of the Fulcher transition is within the $\mathrm{n}=3$ manifold, whereas the upper states of the Werner and Lyman transition are within the $\mathrm{n}=2$ manifold (figure 3 ).

The Fulcher spectrum is mostly undisturbed by other transitions in the wavelength region $600-$ $640 \mathrm{~nm}$ representing the first four diagonal vibrational bands $\left(\mathrm{v}^{\prime}=\mathrm{v}^{\prime \prime}=0,1,2,3\right)$. A very good agreement of the simulation with the measured spectra is obtained for both, the band structure and the spectral emissivity. Due to the fact that this band is used for the analysis of the plasma parameters together with the Balmer line emission using the $\mathrm{CR}$ model one can conclude that the corona approach 
is a good approximation for the Fulcher emission at these plasma parameters. This is consistent with the usage of the vibrational temperature of $\mathrm{T}_{\mathrm{vib}}=3000 \mathrm{~K}$ obtained by the $\mathrm{CR}$ analysis.

Measurement and simulation of the continuum spectrum are shown in figure 6 . The continuum radiation of the $\mathrm{a}-\mathrm{b}$ transition covers the wavelength region from $120-600 \mathrm{~nm}$. The continuum spectrum is strongly overlapped for wavelengths lower than $190 \mathrm{~nm}$ by the continuum emitted from the Lyman band [30] which is dominated by the transition of the v' $=9$ level of the $\mathrm{B}^{1} \Sigma_{\mathrm{u}}^{+}$state into the dissociation limit of the ground state. Above $280 \mathrm{~nm}$ the signal-to-noise ratio of the measured spectrum becomes too small for a reliable analysis. However, the most intense and sensitive part of the spectrum is represented in the wavelength interval $190-280 \mathrm{~nm}$ as explicitly shown in figure 5(b). Roughly one half of the intensity of the continuum radiation is within the selected wavelength interval. Shape and spectral intensity of the simulation are in very good agreement with the measurement using the same plasma parameters as for the simulation of the Fulcher band (table 1). Hence, the corona model is applicable for calculating the continuum radiation and the Fulcher emission, both of them representing transitions in the triplet system, whereas the transitions in the singlet system, the Lyman and the Werner band are underestimated and would require a CR model for which the data base is scarce (see discussion in section 3.3).

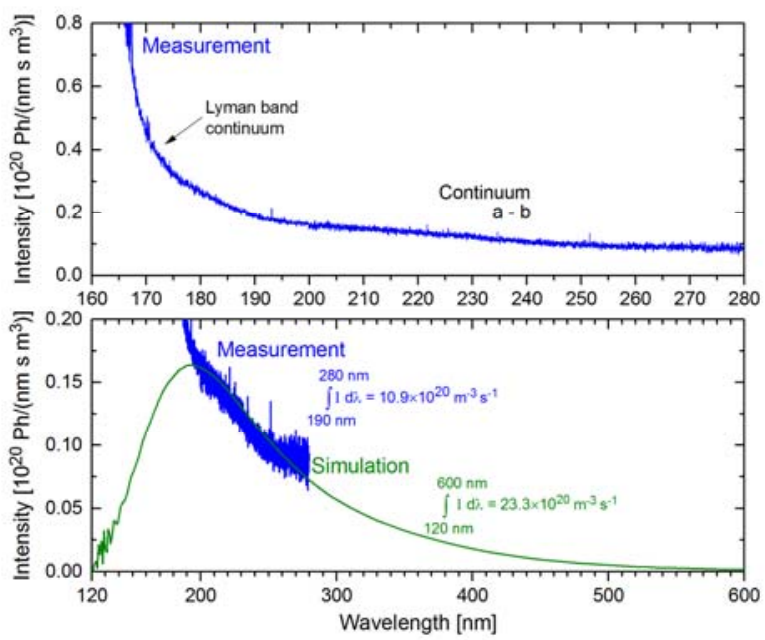

Figure 6. (a) Spectrum of hydrogen plasma at $3 \mathrm{~Pa}$ and $540 \mathrm{~W}$ RF power. The electronic transitions are indicated. (b) Simulation of the continuum radiation ( $\mathrm{a}-\mathrm{b}$ transition) using the corona model and the respective plasma parameters from table 1.

According to the discussion above, the following wavelength intervals are assigned to the individual molecular bands for the evaluation of the radiation quantities from measured data. The wavelength interval from $117-130 \mathrm{~nm}$ is taken to represent the Werner band subtracting out the $\mathrm{L}_{\alpha}$ emission. The interval $130-190 \mathrm{~nm}$ is assigned to the Lyman band; $190-280 \mathrm{~nm}$ are taken for the continuum radiation. The Fulcher band is represented by the wavelength interval between $600-$ $640 \mathrm{~nm}$. The $\mathrm{L}_{\alpha}$ line and the Balmer lines are fitted by a Gaussian profile and then integrated. The simulation of the molecular bands is then used to obtain the scaling factors for the calculation of the full band intensities such that the radiant power or photon fluxes of in the wavelength interval from $80-800 \mathrm{~nm}$ can be derived as well.

\subsection{Plasma parameters from the CR model}

Table 1 summaries the electron density, electron temperature and the atomic-to-molecular density ratio obtained from the analysis of the emissivity of the Balmer lines and the Fulcher transition with the collisional radiative model (see section 3.2. together with the discussion of the uncertainties). The gas temperature and vibrational temperature are derived from the rotational lines and diagonal vibrational transitions of the Fulcher band. The atomic hydrogen density, which is the basis for determining the particle flux, is listed as well. 
Table 1. Plasma parameters of hydrogen plasmas at $540 \mathrm{~W}$ RF power obtained by optical emission spectroscopy.

\begin{tabular}{ccccccc}
\hline Pressure & $\mathrm{T}_{\mathrm{e}}$ & $\mathrm{n}_{\mathrm{e}}$ & $\mathrm{n}_{\mathrm{H}} / \mathrm{n}_{\mathrm{H} 2}$ & $\mathrm{n}_{\mathrm{H}}$ & $\mathrm{T}_{\text {gas }}$ & $\mathrm{T}_{\text {vib }}$ \\
\hline $1 \mathrm{~Pa}$ & $3.2 \mathrm{eV}$ & $0.8 \times 10^{17} \mathrm{~m}^{-3}$ & 0.46 & $5.5 \times 10^{19} \mathrm{~m}^{-3}$ & $600 \mathrm{~K}$ & $3000 \mathrm{~K}$ \\
$3 \mathrm{~Pa}$ & $2.7 \mathrm{eV}$ & $1.3 \times 10^{17} \mathrm{~m}^{-3}$ & 0.57 & $1.7 \times 10^{20} \mathrm{~m}^{-3}$ & $720 \mathrm{~K}$ & $3000 \mathrm{~K}$ \\
$6 \mathrm{~Pa}$ & $2.1 \mathrm{eV}$ & $2.5 \times 10^{17} \mathrm{~m}^{-3}$ & 0.38 & $2.5 \times 10^{20} \mathrm{~m}^{-3}$ & $740 \mathrm{~K}$ & $4000 \mathrm{~K}$
\end{tabular}

At constant RF power the electron temperature decreases with increasing pressure which is the expected general trend of low pressure discharges. The electron density increases with pressure and is in the order of $10^{17} \mathrm{~m}^{-3}$ which is relatively high for hydrogen plasmas at this low power level but the plasma volume is not that large and the corresponding power density results in $0.22 \mathrm{~W} / \mathrm{cm}^{3}$. High dissociation efficiency is observed, i.e. the density ratio of atoms to molecules is in the range of $50 \%$, resulting in atomic densities in the order of $10^{20} \mathrm{~m}^{-3}$ being generally three orders of magnitude higher than the electron density and thus the positive ion density.

\subsection{Radiant power}

The radiant power of the individual molecular band emissions in the respective wavelength intervals and the atomic lines is plotted in figure 7 for the pressure of $3 \mathrm{~Pa}$. The measurements of the molecular band intervals are compared to the simulation based on the corona model using the corresponding plasma parameters (table 1). The excellent agreement between measurement and simulation for the continuum and Fulcher radiation in the respective wavelength intervals underlines again the statement that a ro-vibrationally resolved corona model is a good approximation for transitions in the triplet system. Transitions in the singlet systems are systematically underestimated. The radiation of the atomic lines is calculated with the CR model from which the plasma parameters are obtained resulting intrinsically in a perfect agreement for the Balmer lines. The $\mathrm{L}_{\alpha}$ line shows the largest disagreement between measurement and simulation which is attributed to the fact that analysis is done for optically thin plasmas. In particular resonant atomic lines are very sensitive to self-absorption. This can be considered in CR models by applying escape factors which reduce the transition probability [23]. On the other hand the radiation transfer equation needs to be solved to also correct the measured line emission. Nonetheless it can be stated that the measured $\mathrm{L}_{\alpha}$ line represents a lower bound for its radiated power. In general the same trends are observed for all three pressures. Beside these uncertainties, the benchmark of the models to a variety of molecular and atomic transitions of hydrogen is quite satisfying allowing for predictions of radiation for a wide range in electron density and temperature.

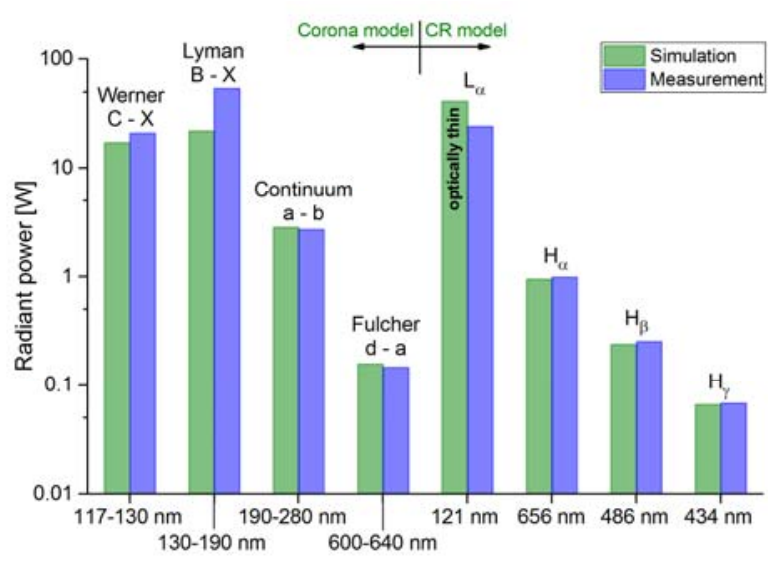

Figure 7. Measured and calculated radiant power for a hydrogen plasma at $3 \mathrm{~Pa}$ and $540 \mathrm{~W} \mathrm{RF}$ power. The calculations are performed with the respective corona models for the molecular bands and the CR model for atomic hydrogen lines using Yacora $\mathrm{H}$ [18]. 
Among the measured molecular and atomic transitions the Lyman band emits in its ascribed wavelength interval the highest radiant power accounting to $54 \mathrm{~W}$ and thus $10 \%$ of the RF power. This is followed by the $13 \mathrm{~nm}$ interval representing the Werner band and the contribution of the $\mathrm{L}_{\alpha}$ line with $21 \mathrm{~W}$ and $24 \mathrm{~W}$ respectively. Although the continuum radiation expands over $90 \mathrm{~nm}$ the radiant power is one order of magnitude lower. The radiant power in the VIS range is low adding up to $1.5 \mathrm{~W}$ in total, i.e. less than $0.3 \%$ of the RF power is emitted in the VIS.

The molecular simulation allows for determining the ratio of a molecular system obtained in the ascribed wavelength interval to the one of total molecular system. For the Werner band the portion is $32 \%$, the Lyman band results in $43 \%$, the continuum in $45 \%$, and the Fulcher band in $50 \%$. Thus, the dominant part of the radiant power is emitted by the Lyman band followed by the Werner band: the measurements results in $75 \mathrm{~W}$ power for the wavelength interval $117-190 \mathrm{~nm}$. The wavelength interval can be extended down to 80 by using the simulation which results in a radiant power of about $140 \mathrm{~W}$ with photon energies of $6.2 \mathrm{eV}(200 \mathrm{~nm})$ up to $15.5 \mathrm{eV}(80 \mathrm{~nm})$.

Table 2 summarizes the results for the three pressures classified into two wavelength intervals: the one representing the VUV emission, i.e. the $\mathrm{L}_{\alpha}$ line, the Lyman and the Werner band as well as the continuum radiation and the other one representing the Balmer lines and the Fulcher transition. Depending on pressure, up to $21 \%$ of the RF power is radiated in the VUV (wavelength interval 117 $280 \mathrm{~nm}$ ), whereas the radiant power in the VIS is negligible low.

Table 2. Radiant power of hydrogen plasmas at $540 \mathrm{~W}$ RF power.

\begin{tabular}{cccc}
\hline Pressure & $1 \mathrm{~Pa}$ & $3 \mathrm{~Pa}$ & $6 \mathrm{~Pa}$ \\
\hline $\mathrm{P}_{\text {rad, } 117-280 \mathrm{~nm}}$ & $50 \mathrm{~W}$ & $101 \mathrm{~W}$ & $111 \mathrm{~W}$ \\
$\mathrm{P}_{\text {rad, 280-800 nm }}$ & $0.6 \mathrm{~W}$ & $1.5 \mathrm{~W}$ & $1.6 \mathrm{~W}$ \\
$\mathrm{P}_{\text {rad, total }} / \mathrm{P}_{\mathrm{RF}}$ & $9.4 \%$ & $19 \%$ & $21 \%$ \\
\hline
\end{tabular}

The results for the radiant power agree well with results from VUV diagnostics of a filamentdriven arc discharge in hydrogen at similar power $(560 \mathrm{~W})$ but lower pressure $(0.18 \mathrm{~Pa})$. According to reference [8] $15 \%$ - 30\% of the discharge power is dissipated via light emission in the wavelength interval $120-250 \mathrm{~nm}$; the total light emission (VUV to visible $80-800 \mathrm{~nm}$ ) has been estimated to be at least $20 \%-40 \%$ of the discharge power.

\subsection{Photon and particle fluxes}

The averaged photon flux in the VUV $(117-280 \mathrm{~nm})$ to a surface at a pressure of $3 \mathrm{~Pa}$ accounts to $5.4 \times 10^{20} \mathrm{Ph} / \mathrm{m}^{2} / \mathrm{s}$ with $57 \%$ being in the wavelength interval of the Lyman band, $21 \%$ in the $\mathrm{L}_{\alpha}$ line and $18 \%$ in the Werner band, whereas the continuum contributes only with $4 \%$. Analogous to the radiant power the measured photon flux of the $\mathrm{L}_{\alpha}$ line represents a lower bound due to opacity effects. The respective flux for the VIS (Balmer lines and Fulcher transition) is $3.4 \times 10^{19} \mathrm{Ph} / \mathrm{m}^{2} / \mathrm{s}$.

The particle fluxes of hydrogen atoms and positive ions to a surface are calculated from the atomic hydrogen density and electron density, respectively (table 1 ). The fluxes are summarized in table 3.

Table 3. Photon and particle fluxes of hydrogen at $540 \mathrm{~W}$ RF power.

\begin{tabular}{cccc}
\hline Pressure & $1 \mathrm{~Pa}$ & $3 \mathrm{~Pa}$ & $6 \mathrm{~Pa}$ \\
\hline$\Gamma_{\text {atoms }}$ & $4.8 \times 10^{22} \mathrm{~m}^{-2} \mathrm{~s}^{-1}$ & $1.7 \times 10^{23} \mathrm{~m}^{-2} \mathrm{~s}^{-1}$ & $2.5 \times 10^{23} \mathrm{~m}^{-2} \mathrm{~s}^{-1}$ \\
$\Gamma_{\text {ions }}$ & $5.0 \times 10^{20} \mathrm{~m}^{-2} \mathrm{~s}^{-1}$ & $7.3 \times 10^{20} \mathrm{~m}^{-2} \mathrm{~s}^{-1}$ & $1.3 \times 10^{21} \mathrm{~m}^{-2} \mathrm{~s}^{-1}$ \\
$\Gamma_{\mathrm{ph}, 117-280 \mathrm{~nm}}$ & $2.7 \times 10^{20} \mathrm{~m}^{-2} \mathrm{~s}^{-1}$ & $5.4 \times 10^{20} \mathrm{~m}^{-2} \mathrm{~s}^{-1}$ & $5.9 \times 10^{20} \mathrm{~m}^{-2} \mathrm{~s}^{-1}$ \\
\hline
\end{tabular}

As well known for low pressure hydrogen plasmas, the atomic hydrogen flux is much higher than the ion flux, being about two orders of magnitude higher in the present discharge. The photon flux in the VUV is comparable to the ion flux being a factor of 1.4-2.1 lower than the ion flux. As for the radiant power, the simulation can be used to derive the photon flux for a wavelength interval starting 
with $80 \mathrm{~nm}$. In this case, the photon flux in the VUV $(80-280 \mathrm{~nm})$ is with a value of $7.8 \times 10^{20} \mathrm{~m}^{-2} \mathrm{~s}^{-1}$ (at $3 \mathrm{~Pa}$ ) slightly higher than the ion flux.

In general, the absolute VUV photon fluxes are in the same order of magnitude as measured in other devices, e.g. [1,11,12]. A direct comparison however is very difficult as the values depend very strongly not only on the plasma parameters and pressure, but also on the plasma geometry. The resonance lines contribute strongly to the photon fluxes onto surfaces, however their opacity plays a very crucial role too. For comparisons of different discharges the measurement device for photon fluxes should be ideally positioned in the close vicinity of the surface. The latter would require the development of a quantitative measurement device for the VUV as such devices do not exist so far. The tendency that the photon fluxes are comparable to the ion fluxes is reflected also in $[11,12]$, which also point out, that in hydrogen admixtures to argon the hydrogen emission, in particular $\mathrm{L}_{\alpha}$, can dominate easily over the argon lines.

\section{Nitrogen}

In nitrogen plasmas, the radiation of the $1^{\text {st }}$ and $2^{\text {nd }}$ positive system of the nitrogen molecule (B-A and $\mathrm{C}-\mathrm{B}$ transition, respectively) dominates in the VIS. The $2^{\text {nd }}$ positive system can be overlapped by the radiation of the $1^{\text {st }}$ negative system of the molecular nitrogen ion $\left(\mathrm{N}_{2}{ }^{+}, \mathrm{B}-\mathrm{X}\right.$ transition) [31, 32]. Atomic nitrogen lines are rarely observed in the VIS, whereas some resonance lines and line ending in the metastable levels are observed below $200 \mathrm{~nm}$ [33]. In the VUV the molecular radiation of the Lyman-Birge-Hopfield system ( $\mathrm{a}-\mathrm{X})$ is dominant.

\subsection{Molecular transitions}

A simplified energy level diagram for molecular nitrogen is shown in figure 8. Beside the electronic states the vibrational levels are indicated together with the optically allowed transitions and the weak intercombination system, the Vegard-Kaplan system. The wavelength regions in which the radiation is obtained is indicated as well. The individual band systems are well separated in a spectrum. Due to the small energy gap between vibrational and rotational levels, vibrational sequences within a band system can overlap, whereas individual rotational lines are strongly overlapped forming the well-known band heads $[31,32]$.

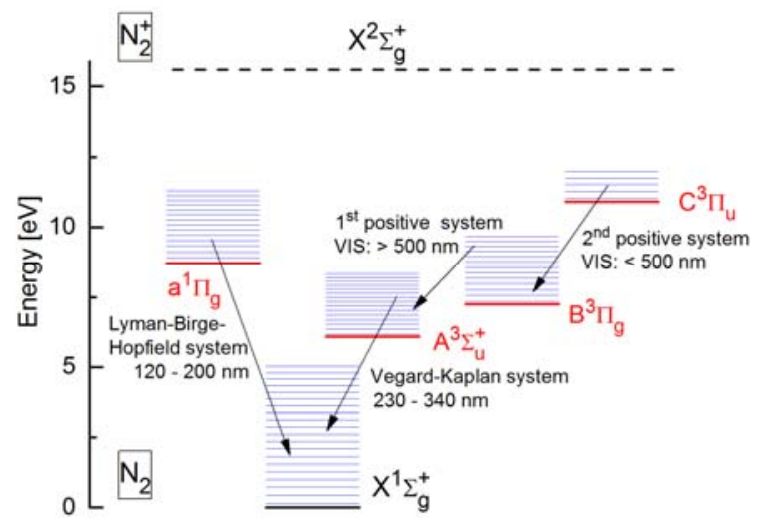

Figure 8. Energy level diagram of the lowest electronic states with the vibrational levels of molecular nitrogen. Optically allowed transitions and the corresponding wavelength region of intense radiation are assigned, together with the weak intercombination system (A-X transition).

\subsection{Example spectra and wavelength regions}

Spectra recorded from the nitrogen plasma at $3 \mathrm{~Pa}$ and $540 \mathrm{~W}$ RF power are plotted in figure 9 . The Lyman-Birge-Hopfield system shows the highest spectral intensity and is partly overlapped by atomic nitrogen lines: the resonance line in the quartet system at $120 \mathrm{~nm}$, the lines in the doublet system ending on the first metastable level $\left({ }^{2} \mathrm{D}\right)$ at $124.3 \mathrm{~nm}$ and $149.3 \mathrm{~nm}$, and on the second metastable level $\left({ }^{2} \mathrm{P}\right) 131.1 \mathrm{~nm}, 131.9 \mathrm{~nm}, 141.2 \mathrm{~nm}$ and $174.3 \mathrm{~nm}$. Although most of the radiation of the Lyman-BirgeHopfield system appears below $190 \mathrm{~nm}$ the wavelength interval of $117-280 \mathrm{~nm}$ is assigned to this system including the atomic lines. This allows for a direct comparison of the nitrogen radiation with the hydrogen radiation assigned to the VUV. 
The $2^{\text {nd }}$ positive system is intense in the UV region and is partly overlapped by the vibrational bands of the $1^{\text {st }}$ negative system of $\mathrm{N}_{2}{ }^{+}$. Vibrational transitions belonging to the same sequence are identified in figure 9 as well as the strong radiation of the $v^{\prime}-v^{\prime \prime}=0-0$ band head of $\mathrm{N}_{2}{ }^{+}$at $391.4 \mathrm{~nm}$. As expected, radiation of the very weak intercombination system, the Vegard-Kaplan system, is not observed. The wavelength interval of $280-420 \mathrm{~nm}$ is assigned to the $2^{\text {nd }}$ positive system. It should be noted that the limits for the wavelength interval are partly determined by the starting and ending wavelength of the high resolution spectrometer. The latter are a compromise between the number of spectra to be taken to cover the wavelength range and the recording of the most intense part of the band systems.
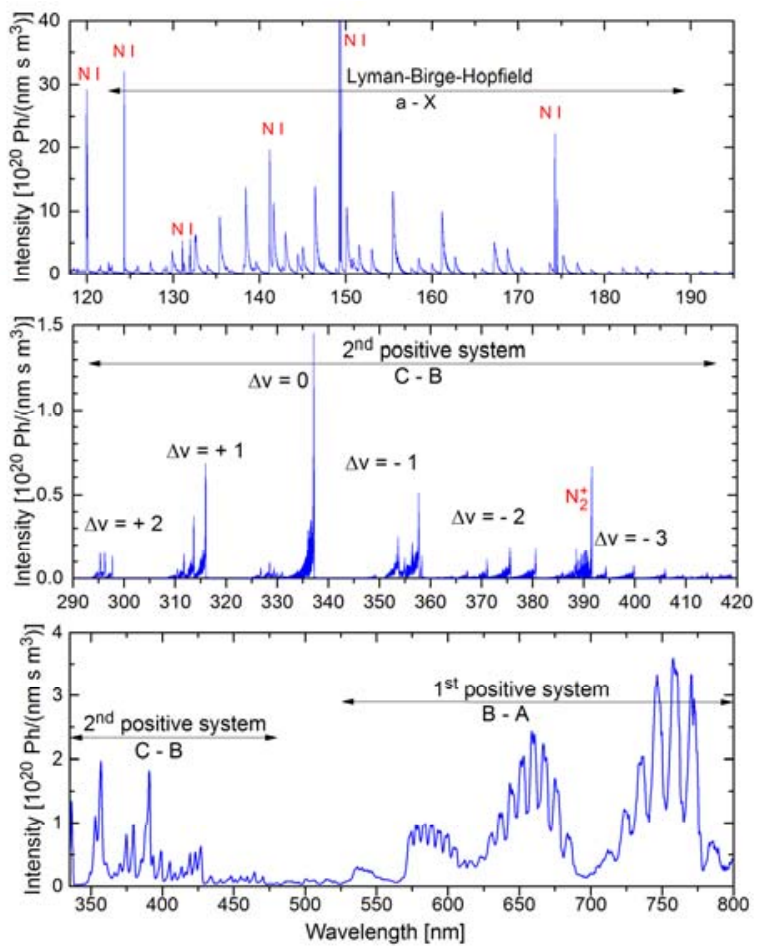

Figure 9. Spectra of the nitrogen plasma at $3 \mathrm{~Pa}$ and $540 \mathrm{~W}$ RF power.

The $2^{\text {nd }}$ positive system and the $1^{\text {st }}$ positive system which are shown in figure $9(\mathrm{c})$ have been recorded with a survey spectrometer and thus with reduced spectral resolution $\left(\Delta \lambda_{\text {FWHM }}=1-2 \mathrm{~nm}\right.$ depending on $\lambda$ ). The $1^{\text {st }}$ positive system is undisturbed and the wavelength interval from $525-$ $800 \mathrm{~nm}$ is assigned to it for the quantitative analysis using data from the calibrated survey spectrometer. Similar to the approach used for the VUV, the wavelength interval of $280-800 \mathrm{~nm}$ (i.e. the both systems together) is assigned to radiation of nitrogen in the VIS, allowing for a direct comparison of the nitrogen radiation with the hydrogen radiation.

Fitting measured vibrational bands of the $2^{\text {nd }}$ positive system with simulations of the molecular band allows for determining the vibrational temperature, the rotational temperature and from that the gas temperature (see $[34,35]$ ). For fitting the rotational distribution, the $v^{\prime}-v^{\prime \prime}=0-2$ transition with the band head at $380 \mathrm{~nm}$ has been used. The best fit is obtained for a rotational temperature of $700 \mathrm{~K}$ and $800 \mathrm{~K}$ for the spectra taken at $1 \mathrm{~Pa}$ and $3 \mathrm{~Pa}$ respectively, with an uncertainty of $\pm 50 \mathrm{~K}$. This corresponds to gas temperatures of $770 \mathrm{~K}(1 \mathrm{~Pa})$ and $880 \mathrm{~K}(3 \mathrm{~Pa})$. The vibrational temperature is determined to be $5000 \mathrm{~K}$ and $7000 \mathrm{~K}$ respectively with an uncertainty of $\pm 500 \mathrm{~K}$. The measurements show that the gas temperatures as well as vibrational temperatures are slightly higher in nitrogen plasmas than the ones obtained in hydrogen plasmas.

\subsection{Radiant power and photon fluxes}

The results for the radiant power and the photon fluxes in the individual wavelength intervals are given in table 4. Operation above $3 \mathrm{~Pa}$ results in an extension of the discharge along the vacuum tubing towards the pumping system which modifies the power deposition and the length of the line of sight for the measurements. For better comparison the measurements have been restricted to $1 \mathrm{~Pa}$ and 
$3 \mathrm{~Pa}$. In nitrogen only $5 \%$ of the RF power is converted to VUV emission, dominated by the resonant a-X transition (Lyman-Birge-Hopfield system) and the atomic lines. In the VIS, 3\% (1 Pa) and 4\% (3 $\mathrm{Pa})$ of the RF power is radiated by the $2^{\text {nd }}$ and $1^{\text {st }}$ positive system. With about $25 \mathrm{~W}$ almost the same power is emitted in the VUV and in the UV-VIS, the latter depending the pressure.

Table 4. Radiant power and photon fluxes of nitrogen plasmas at $540 \mathrm{~W}$ RF power.

\begin{tabular}{ccc}
\hline Pressure & $1 \mathrm{~Pa}$ & $3 \mathrm{~Pa}$ \\
\hline $\mathrm{P}_{\text {rad, } 117-280 \mathrm{~nm}}$ & $26 \mathrm{~W}$ & $27 \mathrm{~W}$ \\
$\mathrm{P}_{\text {rad, } 280-800 \mathrm{~mm}}$ & $18 \mathrm{~W}$ & $25 \mathrm{~W}$ \\
$\mathrm{P}_{\text {rad, total }} / \mathrm{P}_{\mathrm{RF}}$ & $8.1 \%$ & $9.6 \%$ \\
\hdashline$\Gamma_{\mathrm{ph}, 117-280 \mathrm{~mm}}$ & $1.4 \cdot 10^{20} \mathrm{~m}^{-2} \mathrm{~s}^{-1}$ & $1.5 \cdot 10^{20} \mathrm{~m}^{-2} \mathrm{~s}^{-1}$ \\
$\Gamma_{\mathrm{ph}, 280-420 \mathrm{~nm}}$ & $9.1 \cdot 10^{19} \mathrm{~m}^{-2} \mathrm{~s}^{-1}$ & $1.1 \cdot 10^{20} \mathrm{~m}^{-2} \mathrm{~s}^{-1}$ \\
$\Gamma_{\mathrm{ph}, 420-800 \mathrm{~mm}}$ & $2.8 \cdot 10^{20} \mathrm{~m}^{-2} \mathrm{~s}^{-1}$ & $4.2 \cdot 10^{20} \mathrm{~m}^{-2} \mathrm{~s}^{-1}$ \\
\hline
\end{tabular}

The photon fluxes in the UV/VIS are higher than in the VUV and are clearly dominated by the radiation of the $1^{\text {st }}$ positive system. The $2^{\text {nd }}$ positive system shows a comparable photon flux as the VUV emission.

\section{Nitrogen and hydrogen: a comparison}

Admixtures of nitrogen to hydrogen are investigated as well with the composition chosen such that the radiation of the hydrogen atom and the molecule is still present in the spectra. In mixtures radiation of the A-X transition of the NH radical dominates the spectra in the wavelength interval $320-350 \mathrm{~nm}$. Figure 10 shows measured spectra for the mixture $50 \% \mathrm{H}_{2} / 50 \% \mathrm{~N}_{2}$ obtained at the pressure of $1 \mathrm{~Pa}$.
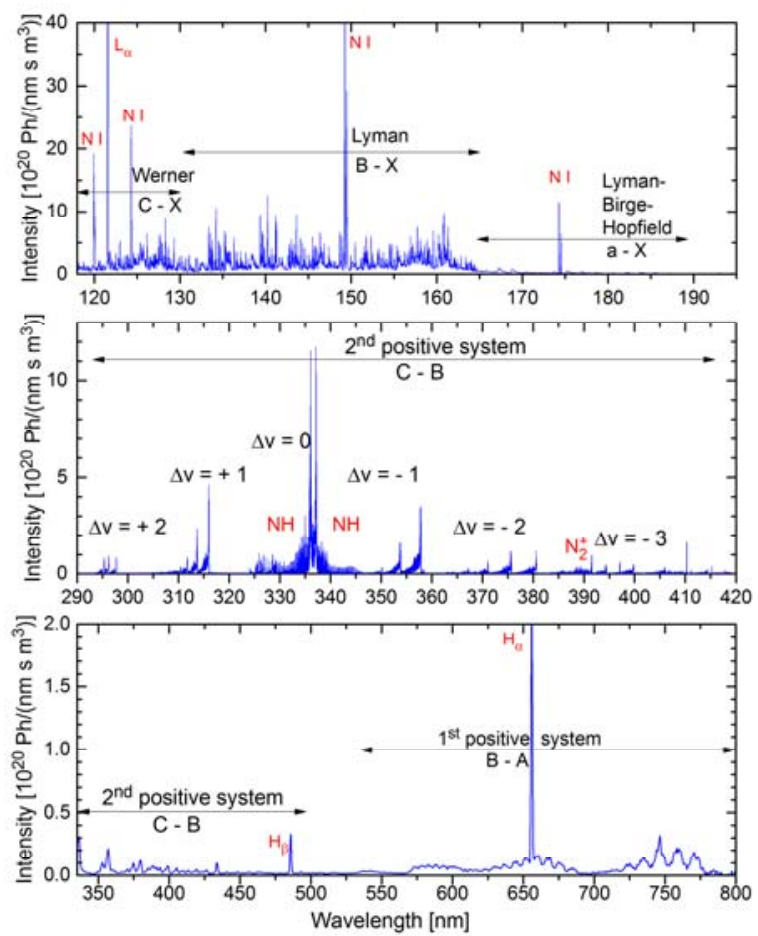

Figure 10. Spectra of the $50 \% \mathrm{H}_{2} / 50 \mathrm{~N}_{2}$ plasma at $3 \mathrm{~Pa}$ and $540 \mathrm{~W}$ RF power.

Figure 11 compares the radiant power of all investigated plasmas with the respective wavelength ranges assigned. 


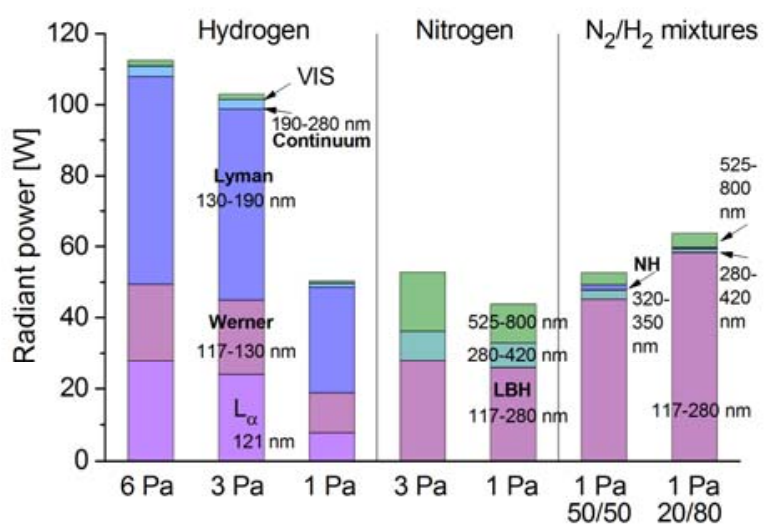

Figure 11. Radiant power for hydrogen and nitrogen plasmas as well as for mixtures at an RF power of $540 \mathrm{~W}$.

The radiant power of hydrogen plasmas is the highest one, dominated by the contribution in the VUV range. In contrast, nitrogen plasmas emit approximately half the radiant power at the same pressure ( $3 \mathrm{~Pa}$ ) and both the VUV and the UV-VIS contribute to almost equal parts. The radiant power in the VUV is only one third of the one in hydrogen. In hydrogen/nitrogen mixtures, the radiant power in the VUV increases with increasing hydrogen content. The UV and the VIS part become less important and the $\mathrm{NH}$ radiation, which is very prominent in the spectra, contributes only with about $1 \mathrm{~W}$ to the total radiant power.

The particle fluxes are presented in figure 12 for plasmas at the same pressure $(1 \mathrm{~Pa})$. The photon fluxes in the VUV clearly dominate in the hydrogen containing plasma, whereas in nitrogen the photon flux in the VIS exceeds the one in the VUV. The UV region becomes relevant only in nitrogen plasmas due to the intense radiation of the $2^{\text {nd }}$ positive system. Compared to the pure gases, different effects change the respective photon fluxes in the mixture. (i) The reduced particle densities of the individual gases in the mixture result in reduced emission. (ii) The plasma parameters like electron density and temperature are modified. The electronic states of molecular nitrogen are energetically below the electronic states of hydrogen such that in admixtures of nitrogen to hydrogen the nitrogen emission dominates, in particular in the VIS and the UV whereas the VUV range is dominated by hydrogen emission. (iii) The direct interaction of hydrogen and nitrogen results in the formation of the $\mathrm{NH}$ molecule which contributes to the UV emission.

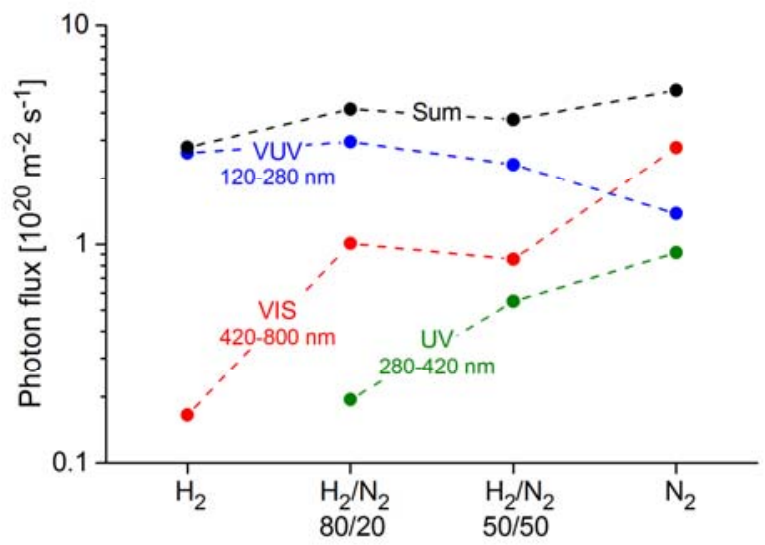

Figure 12. Photon fluxes for hydrogen and nitrogen plasmas as well as for mixtures at $1 \mathrm{~Pa}$ and an $\mathrm{RF}$ power of $540 \mathrm{~W}$.

The dependence of the VUV and UV photon fluxes on the admixture of nitrogen to hydrogen plasmas offers a possibility to tune and even control the respective fluxes for dedicated application processes. 


\section{Conclusions}

The performed intensity calibrated spectroscopic measurements in the wavelength range of $117-800$ $\mathrm{nm}$ allowed for the determination of the radiant power and photon fluxes of hydrogen and nitrogen plasmas in the VUV, the UV and the VIS. Moreover, the good spectral resolution offers the opportunity to assign the quantities to the characteristic molecular emission bands and atomic lines.

In hydrogen discharges up to $21 \%$ of the RF power of $540 \mathrm{~W}$ is measured as radiant power, from which about $110 \mathrm{~W}$ are found in the investigated VUV range $(117 \mathrm{~nm}$ to $280 \mathrm{~nm})$ and only $2 \mathrm{~W}$ or less are radiated in the VIS. The broad spectral emission of the resonant Lyman band in the wavelength range $130-190 \mathrm{~nm}$ contributes to the major part, followed by the Werner band and the $\mathrm{L}_{\alpha}$ line. The continuum emission is of minor importance. Estimations based on simulation of the Lyman and Werner band resulted in $140 \mathrm{~W}$ radiant power at photon energies of $6.2 \mathrm{eV}(200 \mathrm{~nm})$ up to $15.5 \mathrm{eV}$ $(80 \mathrm{~nm})$. In nitrogen plasmas, the radiant power splits almost equally between the VUV and in the UV-VIS reaching up to $27 \mathrm{~W}$ which yields up to $9.6 \%$ of the RF power. Although resonant molecular transitions are also involved in nitrogen plasmas, namely the molecular Lyman-Birge-Hopfield system and some atomic nitrogen lines, the VUV radiation is much weaker than in hydrogen plasmas. In conclusion, this overall remarkable amount of power in the VUV should be considered as loss channel in predictive models for process optimization, especially for hydrogen discharges.

The photon fluxes are in the order of $5 \times 10^{20} \mathrm{~m}^{-2} \mathrm{~s}^{-1}$ and originate, in hydrogen plasmas, from the VUV radiation. About half of the photon flux, i.e. $3 \times 10^{20} \mathrm{~m}^{-2} \mathrm{~s}^{-1}$, is related to the Lyman band representing photons in the energy range of $6.5-9.5 \mathrm{eV}$. About $40 \%$ of the total photon flux, i.e. $2 \times 10^{20} \mathrm{~m}^{-2} \mathrm{~s}^{-1}$, is caused by photons with energies between $9.5 \mathrm{eV}$ and $10.3 \mathrm{eV}$ shared almost equally between the contributions from the $\mathrm{L}_{\alpha}$ line and the investigated interval of the Werner band. In nitrogen low energy photons in the energy range of $1.5-3 \mathrm{eV}$ contribute most, i.e. up to $60 \%$, to the photon flux caused by the strong emission of the $1^{\text {st }}$ positive system in the VIS. The $2^{\text {nd }}$ positive system and the VUV (Lyman-Birge-Hopfield system and some atomic nitrogen lines) contribute with about $20 \%$ each. The relevance of the VUV increases when hydrogen is added to nitrogen plasmas, on cost of the contribution of the UV and VIS. This dependence offers a possibility to tune or even control the respective photon fluxes in plasma processing. Additionally, the characteristic $\mathrm{NH}$ radiation appears in the spectra, with a contribution of $10 \%$ to the photon flux with energies between $3.5 \mathrm{eV}$ and $3.9 \mathrm{eV}$. A comparison of the photon fluxes with the particle fluxes in hydrogen plasmas showed that the photon fluxes are comparable to the ion fluxes, in particular the photon flux in the VUV $(4.4-10.3 \mathrm{eV})$ is $50 \%-70 \%$ of the ion flux. It should be noted that the measurements and the analysis is based on the assumption of optically thin plasmas. In a next step, opacity will be considered. However, it can be already stated that the measured quantities for the resonant $\mathrm{L}_{\alpha}$ line represent a lower bound. In conclusion, in hydrogen and nitrogen plasmas the molecular radiation provides quite high photon fluxes with photons covering a wide energy range. As a consequence photons of such molecular plasmas might play a significant role in the various surface treatment processes.

\section{Acknowledgments}

The authors would like to acknowledge the thorough measurements and analysis of the radial intensity profiles performed by Samet Kurt.

\section{References}

[1] Tian P and Kushner M J 2015 Plasma Sources Sci. Technol. 24034017

[2] Uchida S, Takashima S, Hori M, Fukasawa M, Ohshima K, Nagahata K and Tatsumi T 2008 J. Appl. Phys. 103073303

[3] Lee J and Graves D B 2011 J. Phys. D: Appl. Phys. 44325203

[4] Fukasawa M, Miyawaki Y, Kondo Y, Takeda K, Kondo H, Ishikawa K, Sekine M, Matsugai H, Honda H, Minami M, Uesawa F, Hori M, and Tatsumi T 2012 Jpn. J. Appl. Phys. 51026201

[5] Wertheimer M R, Fozza A C and Holländer A 1999 Nucl. Instr. and Meth. in Phys. Res. B 15165

[6] Titus M J, Graves D B, Yamaguchi Y and Hudson E A 2011 J. Phys. D: Appl. Phys. 44085204 
[7] Hobbs J D and Wesson J A 1967 Plasma Phys. 985

[8] Laulainen J, Kalvas T, Koivisto H, Komppula J, and Tarvainen O 2015 AIP Conf. Proc. 1655020007

[9] Philip N, Saoudi B, Crevier M C, Moisan M, Barbeau J and Pelletier J 2002 IEEE Trans. Plasma Sci. 30 1429

[10] Stapelmann K, Lackmann J W, Buerger I, Bandow J E and Awakowicz P 2014 J. Phys. D: Appl. Phys. 47 085402

[11] Boffard J B, Lin C C, Culver C, Wang S, Wendt A E, Radovanov S and Persing H 2014 J. Vac. Sci. Technol. A 32, 021304

[12] Boffard J B, Lin C C, Culver C, Wang S, Wendt A E, Radovanov S and Persing H 2015 J. Vac. Sci. Technol. A 33, 021306

[13] Komppula J, Tarvainen O, Lätti S, Kalvas T, Koivisto H, Toivanen V and Myllyperkiö P 2013 AIP Conf. Proc. 151566

[14] Mumma M J and Zipf M C 1971 J. Opt. Soc. Am. 6183

[15] Alvarez R, Rodero A and Quintero M C 2002 Spectrochimica Acta Part B: Atomic Spectroscopy 571665

[16] Sharp T E 1971 Atomic Data 2119

[17] Fantz U 2004 Contrib. Plasma Phys. 44508

[18] Wünderlich D, Dietrich S, and Fantz U 2009 J. Quant. Spec. Radiat. Transfer 11062

[19] Wünderlich D and the NNBI Team 2011 Proceedings of the 30th ICPIG Conference, Belfast, Northern Ireland

[20] Fantz U and Wünderlich D 2011 AIP Conference Proceedings 1344204

[21] Fantz U and Wünderlich D 2006 New Journal of Physics 8301

[22] Janev R K, Reiter D and Samm U 2003 Report JUEL-4105

[23] Behringer K and Fantz U 2000 New J. Phys. 2, 23.1

[24] Wünderlich D and Fantz U 2006 Atomic Data and Nuclear Data Tables 92853

[25] Le Roy R 2015 Chemical Physics Research Report CP-668, Available: http://leroy.uwaterloo.ca/programs/ [2016, January 2], University of Waterloo

[26] Celiberto R, Janev R K, Laricchiuta A, Capitelli M, Wadehra J M and Atems D E 2001 At. Data Nucl. Data Tables 77161

[27] Gryzinski M 1965 Phys. Rev. 138 A336

[28] Wünderlich D and Fantz U 2015 XXXII International Conference on Phenomena in Ionized Gases (ICPIG) Iași, Romania

[29] Lavrov B P, Melnikov A S, Käning M and Röpcke J 1999 Phys. Rev. E 593526

[30] Fantz U, Schalk B and Behringer K 2000 New J. Phys. 27.1

[31] Pearse R W B and Gaydon A G 1976 The identification of molecular spectra $4^{\text {th }}$ edition (New York: John Wiley \& Sons, Inc.)

[32] Lofthus A and Krupenie P H 1977 J. Phys. Chem. Ref. Data 6113

[33] Kramida A, Ralchenko Yu, Reader J, and NIST ASD Team 2015 NIST Atomic Spectra Database (ver. 5.3), Available: http://physics.nist.gov/asd [2016, January 2]. National Institute of Standards and Technology, Gaithersburg, MD

[34] Bruggeman P J, Sadeghi N, Schram D C and Linss V 2014 Plasma Sources Sci. Technol. 23023001

[35] Fantz U 2004 Contrib. Plasma Phys. 44508 